\title{
Lidil
}

Revue de linguistique et de didactique des langues

$64 \mid 2021$

Le passif dans la langue parlée

\section{Passifs à l'oral. Le cas du roumain}

Passive Structures in Spoken Language. A Romanian Case Study

\section{Liana Pop}

\section{OpenEdition}

Journals

Édition électronique

URL : https://journals.openedition.org/lidil/9939

DOI : 10.4000/lidil.9939

ISSN : 1960-6052

\section{Éditeur}

UGA Éditions/Université Grenoble Alpes

\section{Édition imprimée}

ISBN : 978-2-37747-315-1

ISSN : $1146-6480$

\section{Référence électronique}

Liana Pop, « Passifs à l'oral. Le cas du roumain », Lidil [En ligne], 64 | 2021, mis en ligne le 01 novembre 2021, consulté le 25 novembre 2021. URL : http://journals.openedition.org/lidil/9939 ; DOI : https:// doi.org/10.4000/lidil.9939

Ce document a été généré automatiquement le 25 novembre 2021.

(C) Lidil 


\title{
Passifs à l'oral. Le cas du roumain
}

\author{
Passive Structures in Spoken Language. A Romanian Case Study
}

\section{Liana Pop}

\section{Introduction}

1 Dans notre démarche, nous donnons d'abord un bref aperçu des formes passives reconnues par les grammairiens roumains, nous essayons de cerner le "sens passif » d'un point de vue graduel, et ce, afin de dénombrer, sur un corpus oral, des formes passives de différents types.

2 Nous avons observé un seul corpus - Interacţiunea verbală în limba română actuală. Corpus (selectiv). Schiţă de tipologie [IVLRA] 'L'interaction verbale en roumain. Corpus (sélectif). Esquisse d'une typologie'-, résultat d'un projet financé conduit à l'Université de Bucarest et à l'Institut de linguistique «Iorgu Iordan - Al. Rosetti » de l'Académie roumaine. L'ouvrage (267 p.) ne reprend qu'une partie des 50 heures d'enregistrement - interactions dialogiques et monologiques classées par type de situation et de fonction ${ }^{1}$. Notre recherche, principalement manuelle, a concerné 8 types de ces textes ${ }^{2}$ et, à ce titre, elle reste relative. De fait, les résultats obtenus ne peuvent pour le moment être que provisoires.

\section{2. État de l'art}

3 Pour le cas du roumain, les grammaires traitent le passif uniquement en termes de « diathèse ${ }^{3}$ ». Ainsi, la grammaire " académique » du roumain (Gramatica limbii române [GALR]) distingue entre constructions («prédicats complexes de type verbal») longues et constructions courtes, suivant la présence de deux agents ou d'un seul, formes classées ensuite en marquées et non marquées (cas du passif lexical, à marque zéro pour ces dernières constructions). Pour les cas marqués :

a. le cas prototypique 4 est construit avec des participes passés (PP) de verbes transitifs précédés de a fi 'être' (Ion este lăudat 'Jean est loué'), ou de ses synonymes relatifs a veni 'venir', a se afla 
'se trouver', a rămâne 'rester', a deveni 'devenir' et a părea 'paraitre', 'sembler' (Ion pare jignit 'Jean semble offensé') ;

b. l'autre cas marqué correspond au passif réfléchi ${ }^{5}$.

4 La construction avec a veni 'venir', moins grammaticalisée, y est qualifiée de populaire, on ne lui reconnait que des "traces de passif», tout comme aux formes contenant le modal a trebui 'falloir', 'devoir', elliptiques du verbe a fi 'être' ${ }^{\prime}$. Le passif prototypique est préféré dans le style recherché, à côté du passif réfléchi dans le style juridicoadministratif (p. 137). Sont invoqués comme passifs lexicaux les participes (adjectivaux, du type om învă Ţat 'homme instruit') et les supins (de + PP : E interzis de utilizat, litt. 'est interdit d'utilisé'; 'c'est interdit d'utiliser') $)^{7}$. En 2005, GALR va introduire pour les constructions passives le critère pragmatique (communicatif) ${ }^{8}$, et, pour certaines expressions, des degrés de passif dues à une transitivité faible, tel l'« élément prédicatif supplémentaire (EPS) ${ }^{9}{ }^{\prime}(E l$ ne-a venit profesor, litt. 'il nous est venu professeur' ; 'il a été nommé professeur dans notre école').

5 Les linguistes roumains ont, de leur côté, à maintes reprises discuté les aspects problématiques des formes passives. Ainsi, Stati (1954) recense les valeurs du participe de formes transitives, intransitives, réflexives ou multiples; Diaconescu (1959) observe les formes du complément d'agent et leurs prépositions introductives dans le roumain ancien, formes génitives ou datives héritées du latin ; Hoarţă-Cărăuşu (2010) constate pour le roumain du $\mathrm{xVI}^{\mathrm{e}}$ siècle une fréquence plus importante des formes réflexivespassives par rapport à aujourd'hui ; Chircu (2010) scrute les structures à deux "éléments prédicatifs supplémentaires" (EPS) successifs et, pour ce qui est de Dragomirescu et Nicolae (2012), ils s'enquièrent, à la suite de plusieurs autres linguistes (Iordan, 1950; Manoliu, 1971; Pană-Dindelegan, 2003), si a veni 'venir' + PP est une construction passive par l'influence de l'italien ou une création roumaine. Enfin, un projet en cours (Boioc Apintei \& Costea, 2020 ; Botez Stănescu \& Dragomirescu, 2020) observe la grammaticalisation de plusieurs verbes de mouvement et de localisation vers des copules ou auxiliaires de passif, ayant des valeurs supplémentaires par rapport à $a f_{i}$ 'être'.

6 À la suite de ces travaux, nous trouvons pertinent de reprendre à GALR les idées d'une gradualité du passif et de traces de passif pour certaines formes moins acceptées, car elles semblent réclamer une définition du sens passif au-delà des structures syntaxiques à la stricte « voix passive ». Pour ce faire, vu le statut souvent fuyant du sens passif, nous considérons appropriée d'adopter pour le passif une perspective en termes de saillance. Ce terme est utilisé en linguistique cognitive pour caractériser la proéminence des éléments dans les énoncés (figures par rapport à un fond ; Langaker, 1987, p. 293), telle, pour le passif, la figure du patient (p. 298). Saillance s'impose aussi à être traité en termes de degrés, comme effet plus ou moins fort des ingrédients prosodiques, lexicaux, syntaxiques, sémantiques et pragmatiques dans les expressions examinées.

Ainsi, afin de situer les nombreuses formes, dites passives, selon leur gradualité, nous proposons dans un premier temps de considérer, à des degrés différents, celles qui :

- sémantiquement, supposent l'existence d'un « patient » - objet direct ;

-grammaticalement, sont à la voix dite "passive»: auxiliaire + participe passé (PP) (+ complément d'agent);

- lexicalement, sont des PP, adjectivisés ou non, et des substantifs déverbaux à sens résultatif ;

- pragmatiquement, thématisent le patient d'un procès ${ }^{10}$. 
En termes graduels, ces particularités donnent, en se combinant, des formes de passif plus ou moins fortes, la forme canonique étant perçue comme la plus saillante, car cumulant à la fois des traits sémantiques (mise en avant d'un patient/objet direct d'un verbe transitif), syntaxiques (structure descriptive périphrastique) et morphologiques (présence d'un PP). Mais, en l'absence du verbe être, par exemple, les formes morphologiques de $\mathrm{PP}^{11}$ semblent se situer différemment du point de vue de leur saillance. Telles :

- les formes de PP disloquées en prédicats secondaires, qui sont plus fortes que celles se présentant sous forme adjectivale (plutôt adjointes), comme : la machine s'est arrêtée [...], bloquée par... ; magasin fréquenté par... ; personne fréquentable ;

- les structures à sens final, subordonnant des infinitifs (un spectacle à voir) ou, en roumain, des supins (uşor de îņ̃eles 'facile à comprendre') laissant le verbe transitif en position subordonnée, plus faible; tout comme les formes intégrées grammaticalement, déverbales à sens statif, issues de dérivations (construction, maquillage ; arrêt, coupe, refus, accord, survol) ou de conversions (lancer, lâcher).

9 Nous observerons plusieurs configurations micro et macrosyntaxiques sous l'angle de la saillance; et ce, tout en tenant compte des effets similaires aux exemples construits ci-dessous, qui montrent que :

a. en termes cognitifs, les formes nominales déverbales sont moins fortes (statiques, non actives, réifiées) que les formes verbales (cf. Langaker, 1987, 2006): Sa condamnation serait une erreur;

b. en termes cognitifs, les formes verbales en positions frontales (intégrées ou disjointes) sont les informations les plus saillantes (focalisées) : Condamné, il irait en prison. Le condamner serait une erreur ;

c. en termes pragmatiques/discursifs, les positions disloquées sont perçues comme fortes aussi bien pour le patient (thématisations) que pour le résultat de l'action (PP fonctionnant, généralement, comme prédications secondes): Lui, condamné, je suis sceptique. En termes grammaticaux, cela correspond à la dichotomie disjoint vs adjoint ;

d. en termes de constructions grammaticales, les positions subordonnées seront perçues comme plus faibles que les positions régentes, plus fortes: On a condamné un innocent $v$ s Il est bon à condamner;

e. en termes de constructions grammaticales, les formes personnelles seraient plus fortes que les formes impersonnelles (passif personnel vs passif réfléchi impersonnel) : Des gens comme lui sont condamnés vs Des gens comme lui se condamnent;

f. en termes sémantiques, les PP seraient plus forts par leur sens résultatif; de même, les formes décrites par rapport aux formes montrées (inférentielles) des structures nominales: Il a été condamné à 10 ans de prison vs La personne condamnée est innocente.

10 Notons que la forme universellement reconnue par les grammairiens comme canonique est une forme descriptive, analytique, avec le verbe être, qui « explicite» l'inversion des rôles dans la configuration [agent-action-patient], les autres formes restant des représentants plus ou moins éloignés du prototype grammatical. Or, pour ces formes moins prototypiques, ce sont les critères sémantiques ou pragmatiques qui ont joué en faveur de leur classement comme plus ou moins " passives $»^{12}$.

11 On verra ci-dessous la richesse des formes à sens passif qui se relayent dans la dynamique du discours, formes fortes ou faibles, en fonction des stratégies de saillance communicative. 


\section{Formes passives dans le discours oral roumain}

Dans le corpus, nous avons retenu des occurrences de passifs canoniques et moins canoniques ${ }^{13}$. Si certaines peuvent facilement apparaitre aussi bien à l'écrit qu'à l'oral, d'autres formes semblent des configurations discursives notamment du roumain parlé.

Seront d'abord prises en considération :

- pour les formes enregistrées dans les grammaires : les formes prototypiques, réfléchies et les passifs lexicaux;

- pour les formes non enregistrées dans les grammaires: des configurations à structures topicalisées, les formes incorporées, elliptiques et en cumul.

\subsection{Formes enregistrées dans les grammaires}

Il s'agit des formes prototypiques avec a fi 'être', du passif réfléchi et du passif lexical. Nous les citons ici dans des énoncés isolés (plus proches des formes déjà reconnues), mais, surtout, dans leur dynamique discursive à l'oral, moins prise en compte par les grammairiens roumains.

\subsubsection{Formes prototypiques}

15

Notons d'abord, deux occurrences prototypiques avec a fi 'être' - en (1) et (2) - et une forme moins prototypique en (3), avec a rămâne 'rester' :

(1)

C: majoritatea spitalelor sînt Ţinute de biserică (237)

‘la plupart des hôpitaux sont tenus par l'église' ${ }^{14}$

(2)

aş dori să vă întreb $\uparrow$ de la /bibisi/. ă: aş dori să vă întreb ă: în CE departamente $\uparrow$ sau în CE sectoare: ă: sunt angajate [...] acele persoane ă: care fac obiectul: diferendumului (156)

'je voudrais vous demander de BBC euh je voudrais vous demander euh dans quel département ou dans quels secteurs euh sont embauchées ces personnes euh qui font l'objet du différend'

(3)

federaţia NU POAte \# să rămînă neconDU:să $\uparrow$ (192)

'la fédération ne peut pas rester non dirigée'

En (4), le verbe prototypique a fi 'être' alterne avec a rămâne 'rester', dans une dynamique discursive fréquente à l'oral :

(4)

A: şi aş dori să trimit u:n $\downarrow$ mesa:j $\downarrow$ pe un mobil $\downarrow$ în franţa. $<_{R}$ şi: aş vrea să ştiu dacă pot să-l trimit $\uparrow$ pentru că am încercat să-l trimit $\uparrow$ şi mesajul a fost stocat .

B: a rămas stocat (196)

'A : je voudrais envoyer un message sur un mobile en France et je voudrais savoir si je peux l'envoyer parce que j'ai essayé de l'envoyer et le message a été stocké.

B : est resté stocké" 
Ce type d'alternance est intéressant à l'oral et a été identifié par BlancheBenveniste (1990) en tant qu'expressions concurrentielles de la valeur passive (p. 52), ou utilisation des multiplies formulations par les locuteurs, comme :

(5)

ça a été imprimé comme ça on a imprimé ça

c'est réfléchi par un miroir à 45 degrés qui réfléchit

ça m'a choqué oui -j'ai vraiment été choqué de de voir cette chose-là

on peut le dessiner ça se dessine comme quand on dessine l'anse d'un panier (p. 53)

Récemment, Druetta (2020) signale lui aussi la tendance des locuteurs à reformuler plusieurs fois l'information: formes de passif canonique reprises par des équivalents actifs, dans un va-et-vient de points de vue visant respectivement le résultat et le procès $^{15}$, comme dans les exemples (6) et (7) que nous lui empruntons :

(6) les vaches vous les avez $\left.\right|_{-} \mid$ah elles sont alpées maintenant $\left.\right|_{-} \mid$et pis comment ça se passe au niveau des classes vous les alpez où

(7)

pis là je suis tombé sur deux Valaisannes qu'avaient _ _ trois semaines de vacances | _ | qu'avaient tout organisé qu'avaient tout planifié qu'avaient tout | _ ouais c'était | _ |

c'était bien carré c'était bien organisé et tout quoi (p. 156)

D'autres occurrences alternent les temps verbaux du même verbe passif, preuve d'un changement de point de vue chez les locuteurs, comme en (8):

(8)

CC: şi a fost publicată

MC: mo- în monitorul oficial este publicată pe ziua de zece ianuarie (154)

'CC : et elle a été publiée

MC : mo- dans le moniteur officiel [elle] est publiée le dix janvier'

ou choisissent une stratégie définitoire, comme en (9):

(9)

MC: îmi $\uparrow$ îmi $\uparrow$ îmi spuneţi ă: nişte lucruri care adică ă: ă: ă: îi numiţi sindicat pe unul care nu este nu e legal constituit. nu este înregistrat la tribunal $\uparrow$ (157)

'MC : [vous] me me me dites euh des choses qui c'est-à-dire euh euh euh vous appelez syndicat quelque chose qui n'est n'est pas légalement constitué n'est pas enregistré au tribunal'

Nous avons également retenu des reformulations par un marqueur définitoire spécifique à l'oral (deci 'donc') introduisant une reprise nominale: accesul 'l'accès' (en 10), une définition en progression, collaborative, pour șosea în câmp 'chaussé en pleine campagne' (en 11), et celle de dumnezeu necunoscut 'dieu inconnu' (en 12) :

(10)

intrarea pe internet fără să mai fii legat de un fir $\downarrow$ \# de un telefon $\downarrow$ \# de ceva $\downarrow$ deci accesul liber pe internet> (209)

'l'entrée sur internet sans plus être lié par un fil de téléphone par quelque chose donc l'accès libre sur internet'

A: e asfalt.

B: da $\downarrow$ dar întuNE::ric. nu e: $\uparrow$ deci nu e SAT $\uparrow$ cîmp cîmp cîmp $\downarrow$

A: o şosea: în [cîmp (46) 


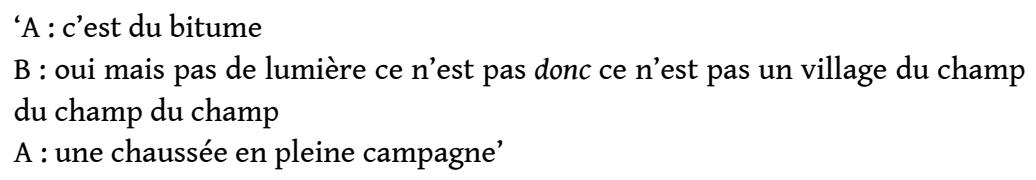

(12)

privIND LOcurile VOAstre de închinARE $\uparrow$ am aflat ŞI un alta:r $\uparrow$ pe care era scris DUMNEZEULUI NECUNOSCUT. $\downarrow$ \# DEci $\uparrow$ pe cel pe care VOI $\downarrow$ NECUnoscîndu-L $\downarrow$ îl CINSTIŢI $\uparrow(251)$

'regardant vos lieux de culte j'ai aussi trouvé un autel sur lequel était écrit au dieu inconnu donc celui que vous ne le connaissant pas vous l'honorez'

Enfin, certaines formes acceptent l'ellipse de la copule a fi 'être', et le cas le plus reconnu dans les grammaires (GALR, p. 135) est la structure avec a trebui 'falloir'. Dans le corpus, nous avons retenu les séquences (13) et (14), la seconde étant un exemple d'alternance discursive de passifs (cercetare finanţată / cercetare care-ar trebui finanţată 'recherche financée / recherche qui devrait [être] financée') :

(13)

trebui [săfie] observat că cel puŢin noi procurorii nu avem de ce să ne plîngem $\downarrow$ (159)

litt. 'il faut [qu'il soit] observé que au moins nous procureurs n'avons pas à nous plaindre'

'il faut observer que au moins nous procureurs nous n'avons pas à nous plaindre'

(14)

CERCEtarea $\downarrow$ din punct de vedere al finanŢării $\uparrow<_{\mathrm{R}}$ am putea s-o împarŢim în: \# cercetare finanŢată DE STAT $\uparrow>$ Şi: cer $_{\perp}$ a: cercetare care-ar TREbui [să fie] finanţată DE CĂTRE marile firme $\downarrow$ \# marii agenŢi economici (207)

'la recherche du point de vue du financement nous pourrions la diviser en recherche financée par l'état et la rech euh recherche qui devrait [être] financée par les grandes entreprises les grands agents économiques'.

\subsubsection{Passifs réfléchis}

Le roumain possède, comme d'autres langues, un passif réfléchi impersonnel en se 'se', classé dans GALR dans les formes marquées; si l'exemple (15) montre un COD postposé, dans les autres, l'objet direct n'est pas précisé. L'équivalent en français du se impersonnel réfléchi est généralement on, comme on peut le voir dans les exemples suivants :

(15)

nu s-au făcut investigaŢii (157)

litt. 'il ne s'est pas fait d'investigations'

'on n'a pas fait d'investigations'

(16)

se investeşte MULT în: $\uparrow$ în acest domeniu? (207)

litt. 'il s'investit beaucoup dans ce domaine ?'

'on investit beaucoup dans ce domaine?' 
(17)

nu se timbrează [suplimentar (101)

litt. 'ne se timbre supplémentaire'

'on ne met pas de timbre supplémentaire'

De (18) à (20), des expressions périphrastiques alternent avec des expressions réfléchies ${ }^{16}$ :

(18)

PN: faptu' că ne-am interesat la tribunal dacă a fost la judecătorie \# dacă a fost innregistrat este un lucru firesc. [...] e firesc să te interesezi dacă s-a înregistrat la judecătoria sectorului şase (158)

'PN : les fait qu'on se soit intéressé au tribunal si [cela] a été au tribunal si [cela] a été enregistré est une chose normale [...] c'est normal qu'on s'intéresse si [cela] s'est enregistré au tribunal du secteur 6'

(19)

A: da $\uparrow$ aşa am primit mesaj de la: centru: de servicii $\downarrow$ că [mesajul] a fost stocat $\uparrow$ şi nu ştiu da-

B: [se poate trimite $\downarrow$ da \# (196)

litt. 'ah oui c'est comme ça que j'ai reçu message du centre de services que [le message] a été stocké et je ne sais pas s-

$B$ : il se peut envoyer oui'

'ah oui c'est comme ça que j'ai reçu le message du centre de services que [le message] a été stocké et je ne sais pas s-

$\mathrm{B}$ : on peut l'envoyer oui'

(20)

A: eu mi-am plăti:t ă ă caZArea şi ma:sa $\uparrow$ şi \# TOŢI ceiLALŢi $\downarrow$ nici măcar [pentru sporTI:VI nu s-a plătit. [...] $<_{\mathrm{L}}$ nici măcar transportu> nu a fost plătit de $<_{\mathrm{R}}$ fedeRAŢie.> (190)

litt. 'A : c'est moi qui me suis payé euh euh le logement et le repas et tous les autres même pas pour les sportifs ne s'est payé [...] ni même le transport n'a été payé par la fédération'

'A : c'est moi qui me suis payé euh euh le logement et le repas et tous les autres même pas pour les sportifs on n'a pas payé [...] ni même le transport n'a été payé par la fédération'

\subsubsection{Passifs lexicaux}

Pour la GALR il s'agit de passifs implicites, "à marque zéro » (p.137). Dans cette catégorie sont inclus des participes adjectivisés et des formes de supin provenant de verbes transitifs, ayant comme trait inhérent (+Passif) et renvoyant le sujet actif en position de complément d'agent : avere bine administrată de moştenitori 'héritage bien administré par les héritiers'; profesor demn de ales director de către colegi 'professeur digne d'[être] élu directeur par ses collègues' (p. 138).

Dans le premier exemple ci-dessous (21), le participe panicat 'paniqué' semble garder, par la présence de la copule a fi 'être', un statut mi-verbal mi-adjectival, mais en (22) legată 'liée' est adjectival, car subordonné directement au substantif cercetare 'recherche' :

(21)

dac-aş vrea să fi:u \# cum să spun eu $\uparrow$ PAniCAT aș spune că [...] (203) 
'si je voulais être comment dire paniqué je dirais que [...]'

(22)

există o cercetare legată $\downarrow<$ J foarte pragmatic $\downarrow$ \# de economia naŢională $\downarrow$ (207)

'il y a une recherche liée très pragmatiquement à l'économie nationale'

En (23), vu leur statut d'expressions passives minimales, plusieurs participes adjectivaux en positions prédicatives peuvent se relayer facilement : les deux premiers, subordonnés à des substantifs (ateu convins 'athée convaincu' et anti mihai întîi afirmat 'anti mihai premier affirmé'), les autres, en double subordination, verbale (a ieşi 'sortir') et nominale (flancat 'flanqué', accordé au masculin singulier). Toute période qui se construit comme ci-dessous a besoin, pour des raisons d'économie, de ces formes brèves à sens passif.

(23)

aŢi fost ă: ateu convins $\downarrow$ aŢi fost ANti mihai întîi \# afirMAT prin fapte $\downarrow$ ieşiŢi acuma în piaţa publică $\uparrow$ flancat \# de cruce şi de coroană $\uparrow$ adică de: prea_sfinţia sa şi de:

[majestatea_sa (147)

'vous avez été athée convaincu vous avez été anti Michel Ier affirmé par des faits vous sortez maintenant sur la place publique flanqué par la croix et par la couronne c'est-à-dire par sa sainteté et par sa majesté'

27 Les formules avec le supin (préposition + PP) ayant un sens de finalité sont fréquentes à l'écrit et, donc, inventoriées par les grammaires roumaines. Dans notre corpus oral, nous en avons extrait :

(24)

e de observat: cu privire la acest aspect $\uparrow[$ [...] o NORmală reticenţă... (159)

litt. 'est d'observé concernant cet aspect une normale réticence...'

'il faut observer/il est à observer...'

(25)

există cerceTAre şi-n domeniul TEXTIL $\downarrow[$ [... care NU este de neglijat (207)

litt. 'existe recherche et dans le domaine textile [...] qui n'est pas de négligé'

'il existe de la recherche aussi dans le domaine textile [...] qui n'est pas à

négliger'

À ces formes de supin correspondent en français des infinitifs à sens passif ou des adjectifs en -ble:

(26)

am şi eu două anunŢuri de făcut (224)

litt. 'j'ai moi aussi deux annonces de fait'

'j'ai moi aussi deux annonces à faire'

(27)

MUzica $\downarrow$ e-aşa ceva de: $\perp$ un obstacol de netrecut între două persoane (222)

litt. 'la musique est comme ça quelque chose de un obstacle de nepassé entre deux personnes'

'la musique est quelque chose comme un obstacle infranchissable entre deux personnes' 


\subsection{Formes non enregistrées dans les grammaires}

Il s'agit de cas où les locuteurs vont promouvoir les patients en positions frontales, au détriment des agents, avec des tours topicalisés/focalisés, typiquement discursifs, et non avec des tours périphrastiques grammaticaux ${ }^{17}$.

\subsubsection{Structures topicalisées}

Certaines formes pronominales focalisent le patient à l'accusatif, voix active, comme dans les exemples de (28) à (30), ne faisant ressortir que de possibles candidats au passif. Du fait de rendre saillant le patient, ces formes, frontales ou finales, sont en roumain : des pronoms toniques (précédées de pe litt. 'sur', de 28 à 30) ou accentuées (en 31 et 32); vu leur position secondaire, les formes toniques finales seront néanmoins perçues comme moins fortes :

(28)

putę̧i să-l luaŢi dumneavoastră direct $\downarrow$ dacă vre⿻̧i $\downarrow$ deci pemine nu mă deranjează cu nimic (6)

'vous pouvez le prendre directement si vous voulez donc moi [ça] ne me dérange en rien'

(29)

PE MINE m-a::i__ameninŢat $\downarrow$ (195)

litt. 'moi tu m'as menacé'

'c'est moi que tu as menacé'18

Par rapport aux formes frontales en (28) et (29), la forme finale en (30) est perçue comme moins forte, mais tous ces tours actifs à patient focalisé peuvent se muer en structures passives. Par exemple : pe mine $m$-ai ameninȚat [tu] > eu am fost ameninȚat de tine ('moi tu m'as menacé' > 'j'ai été menacé par toi').

(30)

nu mă ajutaŢi şi pe mine $\uparrow(17)$

litt. 'ne m'aidez et moi'

'vous ne m'aidez pas moi non plus'

Le pronom relatif pe care (litt. 'sur quel(le)' ; 'que') en accusatif est un cas particulier de focalisation du patient en roumain, avec un redoublement clitique, comme en espagnol ou bulgare (cf. Desclés \& Guenchéva, 1993, p. 80). On l'observe dans l'exemple (31) :

(31)

vă: sun în legătură cu oferta pe care mi-aŢi trimis-o: (19)

litt. '[je] vous appelle en liaison avec l'offre que [vous] m'avez envoyée-la'

'je vous appelle en rapport avec l'offre que vous m'avez envoyée'

33 En (32) et (33) sont promus en fonction de sujet les patients des verbes a purta 'porter' ou a se chema 's'appeler'.

(32)

[ASTA se poartă acum $\downarrow$ roşu şi negru. (244)

litt. 'ÇA se porte maintenant rouge et noir'

'c'est CE QUI se porte/CE QU'on porte maintenant le rouge et le noir' 
(33)

B: PANASONIC se cheamă (202)

litt. 'PANASONIC s'appelle'

'[ÇA] s'appelle PANASONIC'

(35)

(36)

EXISTĂ: IDEEA care se vehiculează $\uparrow$ că în jurul preşedintelui româniei este o nebuloasă (147)

litt. 'EXISTE L'IDÉE qui se véhicule qu'autour du président est une nébuleuse' 'UNE IDÉE EXISTE qui est véhiculée que autour du président il y a une nébuleuse'

En(35), une plus évidente dynamique discursive marque par da' 'mais' une rethématisation, et, par l'incise spre exemplu 'par exemple', un développement périodique complexe :

da' LEMNU \# spre exemplu $\downarrow$ NU nu o să se poată încadra la activ fix $\downarrow$ (199)

'mais LE BOIS par exemple ne ne pourra pas se mettre dans les actifs fixes'

À la différence de (35), la promotion de l'objet en (36) se fait sans marqueur, par la seule position frontale, mais un développement discursif interrompt là encore la construction passive par une incise (vedeŢi 'vous voyez').

TRANSFORMĂRILE ASTEA vedeŢi au fost scăPATE la început (101)

'CES TRANSFORMATIONS voyez-vous ont été omises au début'

Dans ces deux derniers exemples, les topicalisations sont moins perçues comme détachements, vu que les objets directs sont simplement promus en position sujet de par leur emploi dans des structures passives.

Par contre, en structures actives, la dislocation est plus fortement perçue comme position macrosyntaxique, car l'objet détaché, de par sa reprise par un pronom, perd sa position grammaticale de sujet, étant pragmatiquement promu en fonction discursive de thème. Ce phénomène, largement expliqué dans Roulet et coll. $(1985 / 1987)^{20}$, et considéré par Pop (2000) comme une conversion pragmatique, montre le détachement d'une fonction grammaticale (celle d'objet) qui, par une " extraposition », acquiert une fonction macrosyntaxique. 


\section{à (39) -, elle est obligatoire en roumain après un topique défini.}

(37)

ACEASTĂ SUMĂ $\uparrow$ trebuie s-ọ plătiŢi inteGRAL $\downarrow$ (117)

litt. 'cette somme il faut [que vous] la payiez intégralement'

'CETTE SOMME vous devez la payer intégralement'

(38)

SAUNA\# ei vin Ţi-ọmontează $\uparrow$ (199)

litt. 'le sauna ils viennent [ils] te le montent'

'le sauna ils viennent ils te l'installent'

(39)

DOUĂ POSIBILITĂŢI. v-a $a_{\perp}$ vi $\underline{\text { le}}-a$ spus şi dînsu. accepta[Ţi (105)

litt. 'deux possibilités vous a vous les a dites et lui [vous] acceptez'

'DEUX POSSIBILITÉs vous a il vous les a dites lui aussi vous acceptez'

41 Par contre, pour des objets directs indéfinis topicalisés, il n'y a pas de reprise pronominale, comme dans (40) et (41) :

(40)

AVANS \# CîT la> sută doriţi să plătiŢi (200)

litt. 'ACOMPTE combien pour cent désirez que payez'

'POUR L'ACOMPTE quel pourcentage vous désirez payer'

(41)

NICI UN PREŢ NICI O OFERTĂ nu mai faci în euro

litt. 'AUCUN PRIX AUCUNE OFFRE [tu] ne fais plus en euros'

'AUCUN PRIX AUCUNE OFFRE ne se font plus en euros'

\subsubsection{Formes incorporées}

42 Comme pour les participes adjectivisés (dits passifs lexicaux), il faudrait reconnaitre aux noms déverbaux des sens passifs implicites, venant de la transitivité de leur verbe d'origine. Ces noms sont fréquents en roumain, sous la forme d'infinitif long du latin (numirea 'la nomination'), ou de supin nominal (răspunsul 'la réponse'), ci-dessous en (43). Ces contractions nominales confèrent des lectures passives aux syntagmes numirea dumneavoastră 'votre nomination' comme 'nomination faite par vous', et, respectivement, răspunsul dumneavoastră 'votre réponse', comme 'réponse donnée par vous' :

(43)

IS: tocmai $\downarrow$ pentru că se ştie că a fost numirea dumneavoastră $\uparrow$ tocmai de-aia văntrebam $\uparrow$ pentru că $<_{\hat{I}}$ NU este> sentimentul meu $\downarrow<<_{j}$ nu-l cunosc $>\downarrow$ dar există aşa un\# o \# un uşor zvon care circulă şi răspunsul dumneavostră [este $\perp$

II: < î eu cred că e cel mai bun ambasador pe care l-a rumit l-a numit românia după decembrie o mie_nouă_sute_optzeci_şi_nouă> (152)

Lidil, 64 | 2021 
'IS : c'est justement parce qu'on sait que c'était votre nomination que je vous demandais parce que ce n'est pas mon sentiment je ne le connais pas mais il existe un certain bruit court et votre réponse est

II : je pense que c'est le meilleur ambassadeur que la Roumanie ait nommé après décembre mille neuf cent quatre-vingt-neuf'

\section{Sublimé dans les formes nominales, le sens passif y est moins évident, mais cette particularité permet d'amples déploiements discursifs contenant des passifs plus faibles :}

(44)

B: bineînteles. cercetarea este întotdeauna o $\operatorname{activ}_{\perp}$ o INVESTIŢ $[I E=$

A: $=[$ pe termen lung da

$+\mathrm{B}$ : Şi-o investiŢie $\downarrow<_{\mathrm{R}}$ o investiŢie pe termen lung $\downarrow$ da' o investiŢie care: CHIAR că cercetarea $a_{\perp}$ eu nu vreau să se ramînă cu impresia FALSĂ care se creează> $\uparrow$ $<_{\mathrm{R}}$ DOAmne:> $\downarrow$ tot ce se cercetează $\downarrow$ se şi realizează. cercetarea ca: orice: \# activitate care este în domeniul necunoscut $\downarrow$ în domeniul noului $\downarrow$ în

A: da

B: poate să aibă şi ratări (208)

'B : bien sûr la recherche est toujours une activ- un investissement

$\mathrm{A}$ : à long terme oui

$\mathrm{B}$ : et un investissement un investissement à long terme oui un investissement qui vraiment car la recherche je ne veux pas qu'on reste avec la fausse impression qui se crée mon dieu tout ce qu'on crée se réalise aussi la recherche comme toute activité qui est dans le domaine de l'inconnu dans le domaine du nouveau dans

A : oui

B : peut avoir aussi des ratés'

Par ailleurs, pour les formes passives réfléchies, inventoriées à la voix réflexive, l'agent est aussi le patient de l'action encodée par le verbe. Vu cette " incorporation » agent/ patient et leurs expressions minimales - pronoms atones-, elles sont en effet faiblement interprétées comme proprement « passives » :

(45)

B: [știi ce m-am întrebat to_timpu? (194)

litt. '[tu] sais ce que m'ai demandé tout temps'

'tu sais ce que je me suis demandé tout le temps'

\subsubsection{Passifs elliptiques}

Quelques constructions périphrastiques peuvent se construire sans le verbe a fi 'être' (on l'a vu avec le verbe a trebui 'falloir' + PP dans les exemples 13 et 14), ou sans le participe passé et même sans aucun des deux. Les structures avec ellipse du participe semblent être des formes exclusives de l'oral, la plupart du temps favorisées par les formes nominales de passif. Se substituant à un participe, le déverbal livrarea 'la livraison', en (46), permet même une double lecture si on concède au verbe a fi 'être' le sens de 'avoir lieu' et on ignore une possible ellipse du verbe a face 'effectuer' au PP :

(46)

LIVRAREA când $v a f i v$ 
litt. 'la livraison quand sera $\sqrt{ }$ '

'la livraison sera [prévue] (pour) quand'

Une forme impersonnelle non réfléchie mérite d'être mentionnée pour le roumain, celle où le verbe est elliptique, à la troisième personne du singulier, sans sujet explicite, comme en (49), où scrie litt. 'écrit' correspond en français à on écrit ou à la forme périphrastique est écrit :

(49)

în statu:t SCRIe:: $\downarrow$ că orice contract şi orice decizies se ia în birou fedeRAL (193)

\section{3. Élaborations passives en cumul}

On a déjà vu que des combinaisons et/ou des alternances de formes passives grammaticales et discursives sont très courantes à l'oral. Ainsi, de nombreux exemples dans le corpus indiquent, dans des élaborations complexes, une large présence du sens passif, à structures micro et macrosyntaxiques (Pop, 2012), comme dans l'exemple (50) :

(50)

n-am fost ceea ce să spunea: ă: oamenii formaţi la şcoala bolşevică $\downarrow$ [...] oamenii $\mathrm{m}$-au considerat tocmai> ca un om desCHIS

‘je n'ai pas été ce qui se disait euh des gens formés à l'école bolchéviste [...] les gens m'ont considéré justement comme une personne ouverte'

51 De multiples passifs se relayent dans ce fragment : se spunea 'se disait', oamenii formaŢi la 'des gens formés à' et om deschis 'personne ouverte', ce dernier interprétable comme adjectif dans le syntagme om deschis, mais aussi, comme élément prédicatif supplémentaire EPS (prédication seconde) par rapport à $m$-au considerat 'm'ont considéré'.

52 L'exemple (51) : 
(51)

(xxx) UN CONTRACT nu se face $\downarrow$ şi se < uită de el aşa> \# Şi: în general $\uparrow$ î: GRIJA ă: faŢă de: acest contract $\downarrow$ şi de toate anexele lui $\downarrow$ sau LIPSA DE GRIJA $\downarrow$ se va reflecta în: nişte plăŢi $\downarrow$ ă: mai mari $\downarrow$ (113)

'UN CONTRAT ne se fait pas et s'oublie comme ça et en général euh L'INTÉRÊT vis-àvis de ce contrat et de toutes ses annexes ou L'ABSENCE D'INTÉRÊT se reflèteront dans des payements euh plus grands'

- un contract nu se face şi se uita de el aşa 'un contrat ne se fait pas et s'oublie comme ça' montre une topicalisation d'objet (apparemment un déverbal) suivie de deux passifs réfléchis ;

- grija 'l'intérêt', lipsa de grijă 'le manque d'intérêt', plăŢi 'payements' sont des déverbaux a sens résultatif topicalisés.

Enfin, en (52), une forme adjectivale (figură necunoscută 'figure inconnue') s'associe à un réfléchi personnel ( $s-a$ afirmat 's'est affirmé'), à une structure périphrastique prototypique ( $a$ fost promovat 'a été promu') et, enfin, à un autre passif réfléchi (s-a vădit 's'est avéré).

(52)

e: pretenţios să caracterizezi un om numai din contacte $\uparrow$ dar: î: orCINE poate vorbi despre hagi pentru că nu este ă:ă: o figură necunoscută [...] el s-a afirmat din FRAgedă tinereţe ca un: talent înnăscut $\uparrow$ a CONfirmat de fiecare dată $\downarrow$ de cînd a pornit de la faru' $\downarrow$ apoi cînd a [...] venit în echipele bucureştene $\downarrow$ cînd a fost promovat în echipa naŢională $\downarrow$ [...] adică în primul rînd este un om cu un talent naTIV $<_{\mathrm{P}}$ deosebit> $\downarrow$ care $s$ - $a$ vădit: în primele sale ă: manifestări $\downarrow$ (160)

'c'est prétentieux de caractériser un homme par ses seuls contacts mais euh n'importe qui peut parler de hagi parce qu'il n'est pas une figure inconnue [...] il s'est affirmée dès sa première jeunesse comme un talent inné il a confirmé dès qu'il a débuté chez faru' puis quand il est venu dans les équipes de bucarest quand il a été promu dans l'équipe nationale [...] c'est-à-dire en premier lieu c'est un homme de talent natif particulier qui s'est avéré dans ses premières manifestations'

54 Nous nous limitons ici à un simple signalement de ces formes en cumul, mais une recherche approfondie sur le choix de ces passifs dans la dynamique discursive reste surement à faire.

\section{Conclusion}

Grâce à l'analyse du corpus, nous avons pointé du doigt plusieurs constructions passives du roumain parlé: des configurations diverses, certaines potentiellement passives, d'autres lexicales, d'autres qui thématisent l'objet ou le résultat, aux niveaux micro et macrosyntaxique. Nous avons voulu identifier des formes autres que canoniques, grammaticalement admises, et en préciser le degré de saillance.

Sur le plan théorique, nous concluons que pour le roumain il était nécessaire d'élargir la notion de passif au-delà des formes grammaticalisées, ce que nous avons fait ici à travers la notion de gradualité. Les exemples glanés dans cette perspective n'étant qu'une première ébauche du sens passif sur un corpus de roumain parlé, il est clair que le débat sémantico-syntaxique sur ces formes reste encore à faire. 


\section{BIBLIOGRAPHIE}

BERRENDONNER, Alain. (1990). Pour une macro-syntaxe. Travaux de linguistique, 21, 25-36.

BLANCHE-BENVENISTE, Claire. (1990). Le français parlé. Études grammaticales. Éditions du Centre national de la recherche scientifique.

Boioc APINTEI, Adnana \& CosteA, ştafania. (2020). When a veni 'come' Becomes a fi 'be'. A veni 'come' as a Copulative Verb in Romanian. Revue roumaine de linguistique, 65(2), 135-144.

BOTEZ STĂNESCU, Mădălina \& DRAGOMIRESCU, Adina. (2020). The Romanian Verbs a rămâne 'remain' and a se afla 'be situated' on the Grammaticalization Scale. Revue roumaine de linguistique, 2 , 145-158.

Bove, Rémy. (2008). Analyse syntaxique automatique de l'oral : étude des disfluences. Informatique et langage (Thèse de doctorat). Université de Provence - Aix-Marseille 1.

CHIRCU, Adrian. (2010). Construcţii complexe cu EPS şi construcţii derivate de la sintagme cu EPS. Dans A. Dragomirescu \& A. Nicolae (dir.), Limba română: controverse, delimitări, noi ipoteze (vol. I, p. 21-26). Editura UniversităŢii Bucureşti.

CREISSELS, Denis. (2001). Remarques sur la notion de passif et l'origine des constructions passives. Linx, 45, 71-82. <https://doi.org/10.4000/linx.772>.

DEsClés, Jean-Pierre \& GuENTCHÉVA, Zlatka. (1993). Le passif dans le système des voix français. Langages, 109, 73-102. <https://doi.org/10.3406/lgge.1993.1092>.

DGLS = BIDU-VRĂNCEANU, Angela, CĂLĂRAşU, Cristina, IONESCU-RUXĂNDOIU, Liliana, MANCAş, Mihaela \& PANĂ-DINDELEGAN, Gabriela. (1997). Dicţionar general de ştiinţe ale limbii [DGSL]. Editura ştiinţifică.

DIACONESCU, Paula. (1959). Exprimarea complementului de agent în limba română. Limba română, $8(2), 3-17$.

DRAGOMIORESCU, Adina \& NicolAE, Alexandru. (2012). Pasivul cu a veni - traducere din italiană sau inovaŢie românească? Limba română, 1, 73-108.

DRUETTA, Ruggero. (2020). Le passif à l'oral. Phénoménologie et propriétés aspectuelles dans OFROM. Studia lingvistica romanica, 4, 150-174.

GALR = Gramatica limbii române II. EnunŢul. (2005). Ed. Academiei Române.

HAMмA, Badreddine. (2015). Agent passif en par et sujet actif : les dessous d'un contraste. RSP, 37, 61-83.

HAMMA, Badreddine, TARDIF, Amélie \& BADIN, Flora. (2017). Le passif à l'oral. <https:// halshs.archives-ouvertes.fr/halshs-01465258v2/document>.

HOARŢĂ-CĂRĂUşU, LuminiŢa. (2010). Un aspect al morfosintaxei textelor religioase din secolul al XVI-lea: construcţia pasivă. Dans A. Gafton, S. Guia \& I. Milica (dir.), Lucrările ConferinŢei NaŢionale "Text şi discurs religios" (2e éd., p. 269-278). Editura UniversităŢii « Alexandru Ioan Cuza ». <www.diacronia.ro/ro/indexing/details/A71/pdf>.

IORDAN, Iorgu. (1950). Note sintactice. Studii şi cercetări lingvistice, 2, 269-279.

LAGAE, Véronique. (2002). Le passif pronominal : une forme complémentaire du passif périphrastique? Cahiers Chronos, 10, 133-149. 
LAMIROY, Béatrice. (1993). Pourquoi il y a deux passifs. Langages, 109, 53-72.

LANGAKER, Ronald W. (1987). Foundations of Cognitive Grammar. Stanford University Press.

MANOLIU, Maria. (1971). Gramatica comparată a limbilor romanice. Editura Didactică şi Pedagogică.

PANĂ-Dindelegan, Gabriela. (2003). Note asupra pasivului. Dans G. Pană-Dindelegan, Elemente de gramatică. Dificultăţi, controverse, noi interpretări (p. 133-139). Humanitas EducaŢional.

Pop, Liana. (2000). Espaces discursifs. Pour une représentation des hétérogénéités discursives. Peeters.

PoP, Liana. (2010). Les modèles linguistiques face à la problématique du discours. Studia

Universitatis Babeş-Bolyai. Series Philologia, 1-2, 123-143.

Pop, Liana. (2012). De l'oral à l'écrit : articulation micro et macro-syntaxique. Dans M. H. Araújo

Carreira (dir.), Les rapports entre l'oral et l'écrit dans les langues romanes, Travaux et documents, 54, $65-93$.

Roulet, Eddy, Auchlin, Antoine, Moeschler, Jacques, Rubattel, Christian \& SCHELling, Marianne. (1985/1987). L'articulation du discours en français contemporain. Peter Lang.

SECRIERU, Mihaela. (2001). Cumul de funcții sintactice ("elementul predicativ suplimentar") în limba română. Editura UniversităȚii « Alexandru Ioan Cuza ».

STATI, Sorin. (1954). Valorile participiului. Dans Gramatica limbii române (vol. 1, p. 318-337). Editura Republicii Populare Române.

\section{Corpus}

IVLRA = IONESCU-RUXĂNDOIU, Liliana (dir.). (2002). Interacţiunea verbală în limba română actuală. Corpus (selectiv). Schiţă de tipologie [IVLRA]. Editura UniversităŢii din Bucureşti.

\section{NOTES}

1. Il est accessible sur Internet à l'adresse : < www.scribd.com/document/50650439/LilianaRuxandoiu-INTERACTIUNEA-VERBALA-IN-LIMBA-ROMANA-ACTUALA>.

2. Précédés de leurs numéros d'ordre dans l'ouvrage : 24. La judecătorie 'Au bureau juridique'; 26. La secția de poliție ‘À la section de police’ ; 30. DiscuȚie cu un funcționar de la electrica 'Discussion avec un employé de l'entreprise d'électricité'; 38. La agenȚia CFR 'À l'agence de la gare'; 42. Jurnaliști de vorbă cu președintele 'Journalistes parlant avec le président' ; 43. ConferinȚă de presă la Palatul Cotroceni 'Conférence de presse au Palais présidentiel' ; 55. Cerere de informații pentru un contract de leasing 'Demande d'informations pour un contrat de leasing' ; 56. Cerere de informaȚii la telefon 'Demande d'informations au téléphone'.

3. «Voix » n'est pas utilisé dans la terminologie du roumain.

4. Les grammairiens roumains préfèrent dire passif prototypique et non passif canonique (vs non canonique).

5. Certains auteurs l'appellent « pronominal » (voir p. ex. Lagae, 2002 ; Lamiroy, 1993).

6. Tels : CratiŢa [...] vine unsă cu unt [...], litt. 'La casserole vient graissée avec du beurre', équivalente de Cratiţa trebuie unsă cu unt, litt. 'La casserole doit [être] graissée...' (GALR, 2005, p. 78).

7. Le roumain détient des formes de supin (verbales et nominales), majoritairement à valeur passive. 
8. "Reorientarea/deplasarea interesului comunicativ de la nominalul-Agent la nominalulPacient» (GALR, 2005, p.140) 'la réorientation/le déplacement de l'intérêt communicatif du nominal-Agent vers le nominal-Patient'.

9. L'élément prédicatif supplémentaire (EPS) est une fonction à double subordination, nominale et verbale (ex. : Soldatul muri chinuit 'Le soldat mourut torturé') (Secrieru, 2001). Si certains linguistes roumains parlent de sa valeur attributive (dite médiée, dans des structures à verbes prédicatifs, autres que le verbe copule a fi 'être'), dans les grammaires françaises attribut est le seul terme utilisé pour tous ces cas de figure.

10. Pană-Dindelegan, sous l'entrée " passif » du DGSL (1997), décline la notion à des niveaux similaires.

11. Appelées « segments » par Hamma et coll. (2017).

12. Notons néanmoins que placer les formes contextualisées sur une échelle graduelle parfaite reste moins évident.

13. Les pages indiquées comme sources après les exemples se rapportent à ce corpus. Nous gardons intactes les transcriptions d'origine, avec des MAJUSCULES pour les segments emphatiques, mais nous avons ajouté: des italiques pour les formes passives, des PETITES MAJUSCULES pour les topicalisations, et des italiques en gras pour les prépositions/locutions prépositives introductives de compléments d'agent ou certains marqueurs discursifs.

14. De manière générale, nous avons fourni nous-même les traductions françaises des exemples, mais pour les cas qui n'ont pas d'équivalent exact dans l'autre langue, nous avons donné une traduction littérale.

15. Ces phénomènes appartiennent à ce que, au sens large, on appelle disfluence en tant que travail de production, terme concurrencé par plus d'une vingtaine d'autres, chez divers auteurs (Bove, 2008, p. 52-53).

16. On reconnait ici la notion de «parcours actanciel » de Druetta (2020) : « Le recours simultané aux deux diathèses dessine un parcours actanciel complet, ce qui fait ressortir la non-correspondance des arguments syntaxiques et des actants sémantiques. Dans la succession actif-passif, la position sujet est en effet saturée tour à tour par l'agent et le patient, d'où l'appellation de "parcours actanciel". » (p. 156-157)

17. Ces «dislocations" appartiennent de plein droit à la problématique discursive (cf. Berrendonner, 1990 ; Blanche-Benveniste, 1990 ; Pop, 2000, 2010).

18. En roumain, le clivage est peu utilisé, on lui préfère les formes pronominales fortes, détachées.

19. On verra plus loin d'autres cas de passifs elliptiques, dans les configurations périphrastiques (verbe a fi 'être' + participe passé PP).

20. Par des exemples comme: De ce livre, je n'ai jamais entendu parler (construction syntaxique en rection); ou Ce livre, je n'en ai jamais entendu parler (ordre discursif).

\section{RÉSUMÉS}

Dans ce travail, nous nous proposons d'observer les différentes manifestations du passif en roumain parlé compte tenu de ce que les grammairiens reconnaissent comme formes passives. Cela a permis d'identifier de nouvelles occurrences spécifiques à l'oral. Après une définition du sens passif dans une perspective graduelle, nous avons soumis à l'observation, un corpus de 
roumain parlé renfermant plusieurs structures passives canoniques et moins canoniques. Cela a permis de dégager une certaine dynamique discursive que nous espérons décrire avec précision : de nombreuses alternances formelles pour les formes canoniques; quelques formes moins canoniques, non reconnues par les grammaires, dont des topicalisations, des formes nominales déverbales, des structures elliptiques et de nombreux cumuls de formes passives.

In this work, we propose to observe the different manifestations of what we can recognize as a passive meaning in spoken Romanian, taking into account what grammarians identify as passive forms. This allowed us to identify new occurrences specific to the spoken language. After a definition of the passive meaning in a gradual perspective, we submitted to observation a corpus of spoken Romanian containing several canonical and less canonical passive structures. This enabled us to discern a certain discourse dynamic that we hope to describe precisely: numerous formal alternations for the canonical forms; a few less canonical forms, not recognized by the grammars, including topicalizations, deverbal nominal forms, elliptical structures and numerous cumulations of passive forms.

\section{INDEX}

Mots-clés : sens passif, gradualité du passif, formes canoniques et non canoniques, structures discursives de l'oral, langue roumaine

Keywords : passive meaning, graduality of passive forms, canonical and non-canonical forms, discursive structures in spoken language, Romanian language

\section{AUTEUR}

\section{LIANA POP}

Université « Babeş-Bolyai » de Cluj-Napoca, Roumanie 